\title{
The 2021 Mathematical Congress of the Americas: July 19-24, 2021 in Buenos Aires, Argentina
}

\section{Susan Friedlander}

\section{MCA 2021}

Mathematical Congress of the Americas
19-24 July 2021

Buenos Aires

\section{Argentina}

http://mca2021.org
Call for special sessions

Plenary speakers Ian Agol, University of Calliomia, Berkeley Carlos Kenig, University of Chicago Alan Sly, Princeton University Claire Voisin, collège de France Julia Chuzhoy, Toyota Technological Institute at Chicago Miguel Walsh, Universidad de Buenos Aires

Figure 1. Poster for MCA 2021.

The goal of the Mathematical Congress of the Americas (MCA) is to internationally highlight the excellence of mathematical achievements in the Americas and foster collaborations among researchers, students, institutions, and mathematical societies in the Americas. We are very happy to bring to your attention the next MCA, which will be held in 2021 in Buenos Aires, and urge you to look at the website www. mca2021. org.

Susan Friedlander is a professor at the University of Southern California and chair of the Executive Committee of the Mathematical Council of the Americas. Her email address is susanfri@usc. edu.

For permission to reprint this article, please contact: reprint-permission aams.org.

DOI: https://dx.doi.org/10.1090/noti2060
The decision to launch these quadrennial congresses was taken in New Orleans in January 2011 at a meeting of the founding mathematical societies: namely, the AMS (US), CMS (Canada), SBM (Brazil), SIAM (US), SMM (Mexico), and UMALCA (Latin America Union). The inaugural MCA was held in the summer of 2013 in Guanajuato, Mexico. It was an important mathematical event that greatly increased communication and cooperation among mathematicians throughout the Americas. The setting of the lovely town of Guanajuato and the gracious hospitality of the Mexican hosts ensured a delightful background for excellent mathematics. There were many special sessions on a wide range of topics and stellar plenary speakers from across the Americas, including James Arthur, Artur Avila, Manjul Bhargava, Luis Caffarelli, and Ingrid Daubechies. The public lecture by the mathematician-magician Persi Diaconis drew an overflow audience of not only mathematicians but also citizens of the town of Guanajuato.

The Canadian mathematical community played host to the 2017 Mathematical Congress of the Americas in Montréal. By all accounts, the event was a great success: close to 1,100 participants, with 300 coming from Canada, 380 from the US, and about 280 from the various countries of Latin America, with the rest being a smattering from around the globe, from countries ranging from Australia to Zambia. The plenary lecturers all gave beautiful and stimulating talks, as did the twenty invited lecturers, speaking in parallel sessions of three. What was truly unusual was the variety and strength of the special sessions: 74 in all, representing an impressive grassroots effort and showing that indeed such a congress contributes to living mathematics. An effort 
was made in these sessions to get a good North-South split, and by and large this was achieved.

As noted, MCA 2017 saw strong participation from Latin America, which is absolutely vital for the strength of such an event, and the various participating institutions and institutes helped greatly in the effort. The role of the AMS, in particular, was crucial in the preliminary organization and hosting the travel funding grant program. The AMS also piloted an NSF grant application, which was used to fund junior participants, as well as contributing some travel grants from its own funds. Additional funds came from the Canadian Mathematical Institutes; the Mexican NSF; the Brazilian Mathematical Society and the Institute for Pure and Applied Math in Rio de Janeiro; and the Argentinian Mathematical Society. All of this was deeply appreciated and helped a great deal.

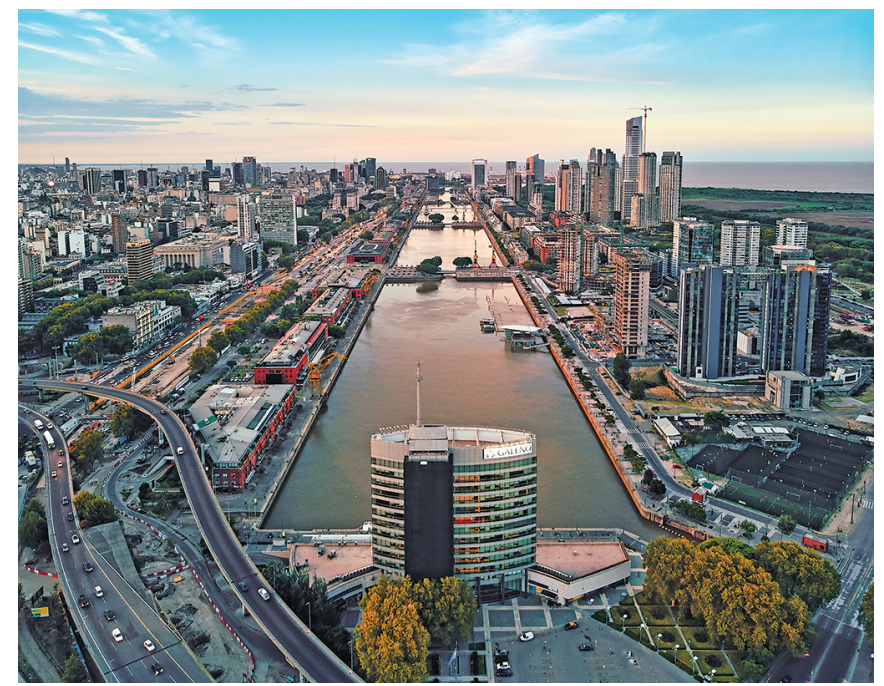

Figure 2. Center of Buenos Aires.

The third MCA will take place in the historic capital city of Argentina and will be hosted by the Departamento de Matemática, Facultad de Ciencias Exactas y Naturales of Universidad de Buenos Aires. We are pleased to report that a large new building on the campus has recently been completed and will provide a spacious and convenient setting for all the activities of the MCA 2021. The scientific program of the congress is well underway with the selection of the plenary and invited speakers. Their selection by the international scientific program committee is based on excellence in research and very good expository skills. These outstanding mathematicians include Ian Agol, Julia Chuzhoy, Carlos Kenig, Alan Sly, Claire Voisin, and Miguel Walsh. We strongly encourage mathematicians from all over the Americas to submit proposals for special sessions following the information found at www.mca2021.org. We seek to host many special sessions on a very broad selection of topics and with diverse participation. The MCA 2017 included 74 such special sessions, and we would be delighted to have a similar number at MCA 2021.
The Mathematical Council of the Americas, which is the organization that oversees the selection of the locations of each congress, established the following mathematical awards to acknowledge accomplishments that are of special relevance to the goals of the MCA:

The MCA Prize: Five prizes of US $\$ 1,000$ each will be awarded to mathematicians who are no more than twelve years past their PhD on July 1, 2021. Eligibility for consideration of nominees requires that they either received their graduate education or currently hold a position in a university or institution in the Americas.

The Americas Prize: One prize of US $\$ 5,000$ will be awarded to an individual or group in recognition of their work to enhance collaboration and research that links mathematicians in countries in the Americas.

The Solomon Lefschetz Medal: Two medals with an award of US $\$ 5,000$ will be given to mathematicians in recognition of their excellence in research and their contributions to the development of mathematics in the Americas.

Nominations for these prizes should be made by email to mca2021. prizes@gmai 1 . com by January 31, 2021.

A number of mathematical societies in the Americas worked together to ensure that funds were available to give travel support to early career mathematicians who wished to attend MCA 2013 and MCA 2017. In particular, the AMS made successful grant applications to the US NSF. We anticipate this will also be the case for MCA 2021. In due course information will be available on www. mca2021 . org detailing how mathematicians in North America, the Caribbean, Central America, and South America can apply for travel funds.

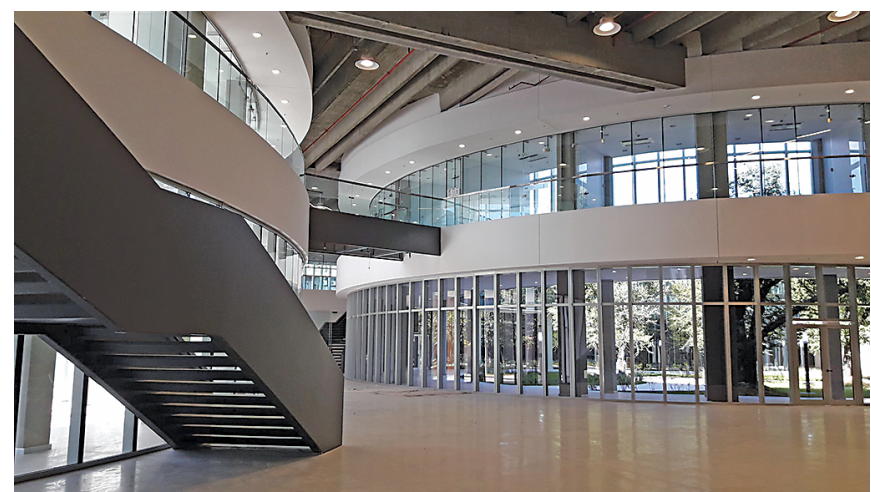

Figure 3.The new building of the Faculty of Science where MCA 2021 will take place.

Argentina has a very strong tradition of mathematical research. Pioneering work in the early 1900 s set the foundation for the development of a wide variety of areas where Argentinian mathematicians have a strong presence. Some Argentinians established major centers in mathematical institutes in Buenos Aires, Cordoba, Santa Fe, Bahia Blanca, and La Plata. Many Argentinian mathematicians are well known for their results, with A. Calderon, L. Caffarelli, and 


\section{COMMUNICATION}

C. Kenig being prominent examples in the field of analysis. The country of MCA 2021 is an excellent site to host a major international mathematical event, both because of its strong culture of mathematics and its very attractive location. We urge our colleagues in all parts of the Americas to place Buenos Aires in July 2021 on their calendars for a very special congress.

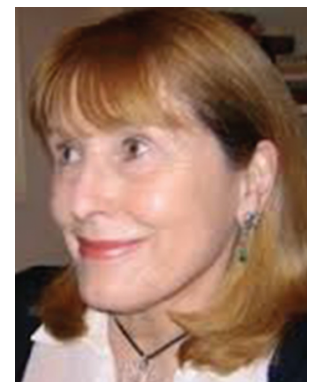

Susan Friedlander

\section{Credits}

Figure 1 is courtesy of Instituto de Investigaciones Matemáticas Luis Santaló.

Figure 2 is by Deensel [CC BY 2.0 (https://creativecommons . org/licenses/by/2.0)] via Wikimedia Commons.

Figure 3 is by Santiago Figueira.

Author photo is courtesy of the author.

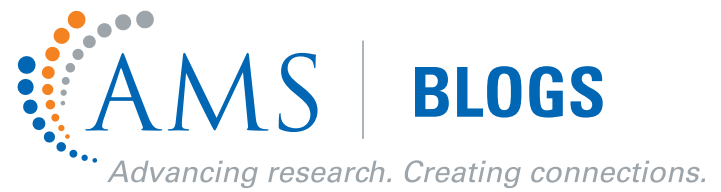

\section{Why navigate your graduate school experience alone?}

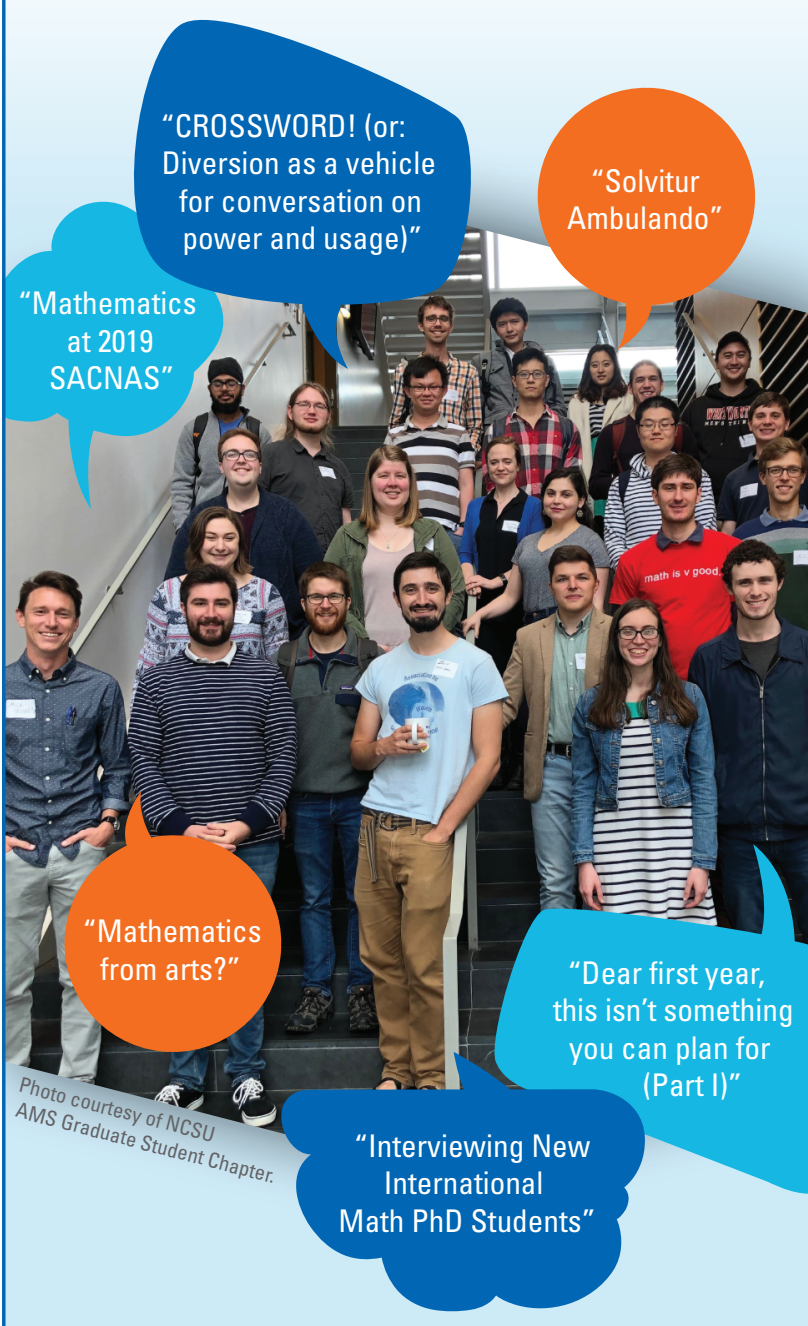

Follow and join the conversation at blogs.ams.org/mathgradblog a blog for and by grad students.

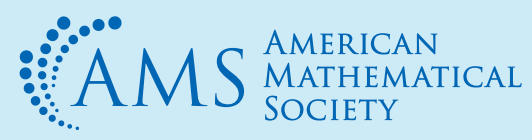

